\title{
The world is not a theorem
}

\author{
Stuart A. Kauffman ${ }^{1}$ and Andrea Roli ${ }^{2,3}$ \\ ${ }^{1}$ Institute for Systems Biology, Seattle, USA \\ ${ }^{2}$ Department of Computer Science and Engineering, Campus of Cesena, Alma Mater Studiorum \\ Università di Bologna \\ ${ }^{3}$ European Centre for Living Technology, Venezia, Italy
}

January 1, 2021

\begin{abstract}
The evolution of the biosphere unfolds as a luxuriant generative process of new living forms and functions. Organisms adapt to their environment, and exploit novel opportunities that are created in this continuous blooming dynamics. Affordances play a fundamental role in the evolution of the biosphere, as they represent the opportunities organisms may choose for achieving their goals, thus actualizing what is in potentia. In this paper we maintain that affordances elude a formalization in mathematical terms: we argue that it is not possible to apply set theory to affordances, therefore we cannot devise a mathematical theory of affordances and the evolution of the biosphere.
\end{abstract}

\section{Prologue}

[L'universo] è scritto in lingua matematica, e $i$ caratteri son triangoli, cerchi, ed altre figure geometriche, senza i quali mezi è impossibile a intenderne umanamente parola; senza questi è un aggirarsi vanamente per un oscuro laberinto. ${ }^{1}$

(Galileo Galilei, Il Saggiatore, Roma (Italy), 1623)

\section{Introduction}

Egyptian papyri dating some 5000 years ago document the use of arithmetic and geometric notions for solving practical problems, such as the need of measuring and subdividing the soil [18]. After thousands of years, three centuries before Christ, we find the most

\footnotetext{
1 "The universe is written in the language of mathematics, and its characters are triangles, circles, and other geometrical figures, without which it is humanly impossible to understand a single word of it; without these, one is wandering around in a dark labyrinth." Translation by S. Drake.
} 
remarkable development of these notions: the Elements, by the Greek Euclid of Alexandria, a prominent and influential deductive theory - queen of the Sciences and true of the world: the language in which the universe is written is made of triangles and circles, as Galileo stated in the XVII century. Nevertheless, an axiomatic and deductive system such as the Elements does not need to describe the world, as shown by non-Euclidian geometries. However, mathematical systems shine as pure crystals and one might expect them to be consistent and complete, as indeed Hilbert did. These hopes have been destroyed by Gödel, who has set the limits of deductive systems. Despite these limits, we currently rely on mathematical models for understanding systems of any sort, conscious of the possible incompleteness of the deductions we make. But there is a kind of incompleteness we have probably overlooked: what can be entailed by a formal system is already contained in it, and we cannot expect to be able to deduce novelty, to entail the becoming of the biosphere.

In this paper we maintain that the flourishing evolution of the universe cannot be captured by a formal mathematical model: the world is not a theorem.

We start by discussing the notion of affordances and the properties of the becoming of the biosphere, in Section 2. Section 3 illustrates the core of our thesis: affordances defeat any sound definition of set, so no set theory, hence no math, can be used to model affordances. Several objections might be raised to argue against our claim: in Section 4 we analyze and answer to the ones we think are the most relevant. Finally, in Section 5, we observe that the essence of our statement can be already found in the late writings by Wittgenstein and we summarize the main points of this paper, emphasizing that, rather than projecting a dark and negative perspective, our claim has a creative and positive potential.

\section{Affordances and the becoming of the biosphere}

The notion of affordance has been originally introduced by Gibson [5] in psychology with the aim of expressing the actions that an object enable to an animal observing it. The concept has been subsequently extended, and it is currently adopted in diverse fields such as biosemiotics, cybernetics and robotics $[2,7]$. In general and abstract terms, we can say that an affordance is "the use of $X$ to accomplish $Y$ ", where $X$ may be an object, a living being, a situation, etc., and $Y$ is in general an action or a behavior that typically leads to a goal. Heritable variations and selection make it possible for organisms to find new, advantageous uses afforded by objects or other organisms. For example, an empty snail shell affords the pagurus a house where it can hide and protect itself from predators, or a colony of bacteria that is evolving in a new environment may discover a more efficient way to get to the food through favorable mutations and selection. In the same way, new organs have emerged in organisms as they afforded opportunities that enhanced their fitness in their environment. By organism we mean a Kantian whole: an organized being having the property that the parts exist for and by means of the whole $[9,13]$ that senses the world, chooses between what is good or bad for it, and acts. In general, affordances open possibilities either by some adaptive (possibly evolutionary) advantage, such as the case of Darwinian preadaptations 
(e.g. the evolution of the swim bladder [11]) or because of a choice of the organism-which recognizes an advantageous opportunity and acts consequently.

An affordance is not a property of the object or the environment only, but it is an entangled property of both the object and the agent. ${ }^{2}$ In fact, affordances cannot be defined in a non-circular way. Niches are prominent example of this, as they constitute the subjective world of an organism [25].

To discuss in more detail the properties of affordances relevant for our argument, we make a brief excursus in a futuristic scenario. Let us imagine a robotic environment, maybe on a rocky extrasolar planet, where robots extract minerals, work in factories producing several materials and goods; moreover, by executing specific programs, they build and assemble the components to produce new robots. One day a robot, failing to decrease its speed fast enough after a declivity, accidentally stumbles onto a sharp and pointed rock and gets a dent on its aluminium side. ${ }^{3}$ The rock turns out to be made of obsidian, very useful to the robots' community. This accident has no consequences on robot's functions, but opens up the possibility for the robot to detect rocks containing obsidian, which protrudes from the rock trunk, because the dent fits rather well with obsidian extrusions. The dent affords the robot to identify obsidian rocks more efficiently and becomes then an "obsidian detector" for the robot. Note that this sensor has a meaning for this robot and in this particular environmental niche.

Let us imagine that a new robot is built with an error in the assemblage procedure and gets a bump on its aluminium side. ${ }^{4}$ We now have also a "dent sensor". This bump enables the robot to detect dents in other robots and then exploit this information to identify the robot that is more efficient in finding obsidian and follow it. Should the robot with the bump be specialized to work to shape obsidian, then the dent in the first robot may serve also as a "bump detector". Therefore, the two special robots can recognize each other and form a specialized team performing extraction and processing of obsidian more efficiently. ${ }^{5}$

Bumps and dents, and bumps and dents detectors, did not exist prior to the accidents and they could not even be predicted. The accidental sliding of a wheel and the impurity in the aluminium opened up the possibility of bumps and dents. These accidents enabled new forms of behavior and cooperation in the niche where the robots operate: affordances create new affordances. It is also worth observing that bumps and dents, along with their detectors, have acquired a meaning because they are useful for a robot to do something, to be more efficient in accomplishing a task. They have a meaning only in this specific niche and for these robots. Now that they have a meaning, the robots can name and use them inside their control programs, which we assume to be adaptive to some extent. Observe

\footnotetext{
${ }^{2}$ By "agent" we mean the subject w.r.t. which the affordance gets a meaning. For example, an agent can be an organism, an artificial system or even an inanimate object.

${ }^{3}$ The cause of this failure is that a wheel slid on the flat ground instead of rotating.

${ }^{4}$ The cause of the error is an impurity in the aluminium sheet that deceptively recursively attracted the accumulation of more aluminum in a small area of the chassis.

${ }^{5}$ For the roboticists wandering how is it possible that the robots actually exploit bumps and dents without having specific pre-designed sensors, we observe that bumps and dents can lock the rotation of the robot and this event can be detected by a specific sensory-motor pattern [22].
} 
that this meaning-making event creates information, which can subsequently be used inside a formal system. A new variable, grounded to a robot's embodied experience [6, 21], is created and added to the program. First comes semantics, then syntax. This kind of information is substantially different from the one which is the subject of information theory, where there is no creation of information but only symbols transmission and loss: "Frequently the messages have meaning; that is they refer to or are correlated according to some system with certain physical or conceptual entities. These semantic aspects of communication are irrelevant to the engineering problem." 6

Let us now imagine that we want to equip the robots with a program that can predict affordances and the emergence of new uses of objects. One might be tempted to think that once a formal model of objects and relations is defined, e.g. by means of a suitable logic language, it would in principle be possible to entail the evolution of this formalized multi-robot colony, at least in terms of the most likely scenarios. This is the same as trying to predict the evolution of the biosphere, but there are no entailing laws for the becoming of the biosphere $[11,12,16]$. In the next section we detail this statement extending it to a stronger claim: not only there are no entailing laws for the evolution of the biosphere, but affordances and the evolution of the biosphere - and all the systems with analogous properties, such as the technological market - are inherently not mathematizable.

\section{Set theory and the limits of mathematical modeling of affordances}

The blooming evolution of the biosphere is characterized by the emergence of new organisms, which in turn evolve new functions. New organisms and new functions open further possibilities for adaptation and evolution: the biosphere evolves by expanding towards the adjacent possible [10]. Affordances play a fundamental role in this evolution, as they represent opportunities for the organisms: they enable the development of new functions, and the emergence of new niches and new organisms.

The notion of affordance is not restricted to the biosphere, but we also find it in other contexts, such as the technological market, where new technologies enable the emergence of new functions, which in turn make it possible to create further new technological devices and methods. A typical example of affordances is that of the uses of an engine block: obviously it can be used as propulsive component in a car, it is used both as engine and chassis for tractors, we can crack open coconuts on one of its corners, its cylinder bores can host bottles of wine, the engine block can be used also as a paper weight, and so on. ${ }^{7}$

How long is this list? One might answer that the list is extremely long, but finite or someone else might argue that it is infinite. We actually argue that the affordances of an object are indefinite. We first observe that the affordances of an object are not an intrinsic

\footnotetext{
${ }^{6}$ Quoted from [24], italics in the original text.

${ }^{7}$ See also a discussion on the possible uses of a screwdriver [11].
} 
property of the object itself, but they also depend on the agent-which exploits them by heritable variations and selection or sees them in the object.

As already stated, the object affords an action to an agent because it enables a possibility for achieving a goal or doing something better, in a more efficient or effective way. The affordances of an object depend upon the individual goals of the agent: the possible uses of an engine block depend on the perspective from which that tool is seen by the agent. They depend upon the meanings that the agent attributes to the engine block. As another example, let's take the swim bladder, which is believed to have evolved from the lungs of lung fish: water went into a lung, now containing both air and water, and afforded a new possible use becoming the swim bladder [12]. New functions come to exist because they mean something to the agent, they are useful for something [2]. Montévil nicely explains this aspect discussing the notion of novelty [19]: as for the books in the Library of Babel by Borges [1], where all the 410 pages long books composed of all the possible combinations of characters are stored, what distinguishes a book from a printed random sequence of letters is the meaning that the reader gives to the sequence of letters; the meaning depends upon the experience, the history of the reader. In addition, the experience of the reader in turn depends upon the environment in which they live. In other terms, affordances cannot be defined non-circularly. For example, technological devices are sold by adapting them to the specific niche corresponding to preferences of the customers. And in biology, the mixed microbial communities living in the gut of animals are reciprocally adapted, and their composition and dynamics also depends on the characteristics of the host [14]. Furthermore, the universe is non-ergodic [10, 16, 11], as its dynamics does not visit all the possible states, as opposed to ergodic systems, which visit all their possible states in a time vastly longer than the present lifetime of the universe. In fact, the universe visits just a tiny fraction of its possible states; let's think for example of the molecules that have been formed vs. the ones that could have come to exist. Non-ergodicity prevents us from defining a phase space that can contain all the relevant variables - and thus define equations and study the trajectories of the objects we are interested in-because we don't even know what the relevant variables are. This holds also in the case of the biosphere, where the expansion of Hilbert space cannot be predicted within a universe lifetime time horizon [3].

Therefore, the uses of an object, i.e. its affordances, are not finite nor infinite but rather indefinite, and there are no deductive rules to list them starting from an initial one nor to deduce some uses of an object from the uses of another object. Since new uses arise all the time by Darwinian preadaptation - or exaptation-evolution cannot be entailed $[11,12,16]$.

A dramatic implication of this statement is that set theory cannot be applied in modeling affordances, because the fact that the affordances of an object are indefinite defeats any definition of set. The main reason for this indefiniteness is that the collection of uses of $X$, alone or with other objects, is constantly changing, therefore there is no way to express the property of $X$ belonging to a set once and for all. Let us take, for example, the axiom of extensionality, which is one of the axioms that define sets [8] and states that "If two 
sets $A$ and $B$ have the same elements, then they are equal". Let's assume that we want to denote by $A$ and $B$ the collections of affordances of objects $X$ and $Y$, respectively, and suppose that we can identify a specific use of $X$ that we call $u$; therefore, $u \in A$. In virtue of the indefiniteness of affordances, it is not possible to assert the truth of $u \notin B$, i.e. it is not possible to determine that $u$ is not and will never be a use of $Y$.

Also the axiom of choice [20], which is introduced whenever a choice function cannot be defined, cannot be applied. One might object that this could be a situation analogous to real numbers, for which the axiom of choice and set theory hold. But we observe that real numbers may be given as sequences of symbols from a finite alphabet, i.e. we know the symbols composing these infinite strings. In the case of new affordances coming to exist over time like the uses of the engine block alone or with other things, the uses have not yet come to existence, therefore the syntactic names have not come to existence, so we do not know the syntactic symbols we would use to name the elements of the set of uses of an engine block. The evolution of the biosphere is indeed characterized by the appearance of new functions and new organisms. The human heart is an affordance; it affords a means to pump blood to the body. The loop of Henle affords a means to concentrate urine in the kidney [17]. And, neither of these affordances existed on the earth two billion years ago, so we cannot do set theory with respect to them.

We are not claiming that there is no possibility whatsoever to model affordances, but that it is possible only in the syntactic frame already defined for the model we have built. Coming to the robotics scenario, robots cannot predict a new use not already deductively present, therefore the flourishing evolution of the biosphere is beyond math because the notion of set, and consequently all the formalisms that derive from it, does not hold in this case. Of course, the possibility of making theories of the physical world still holds, but it is bounded by the objects and relations we decide to include; in other words, we see what is relevant, we name it and we put it in the model, which is a syntactic formalization of the piece of reality for which we want a theory [15]. Nevertheless, we are maintaining that any attempt of modeling a system encompassing affordances or any theory trying to capture novelty and open-ended evolution would be inherently flawed [4, 12, 13, 23].

\section{Possible objections}

We understand that our claim might sound either trivial or provoking, or both. In the following we anticipate what we believe are the main objections that may possibly be raised against our argument.

Objection If we could find a suitable level of description of the universe, then affordances could be modeled as trajectories in this huge space. The relevant trajectories would be extremely rare in this space, as some specific given real numbers are among reals; this set might even have measure zero, but still exist. And if the set of uses of an object is uncountable, then we can anyway apply set theory and, in particular, the axiom of choice 
for picking elements from the sets, as we do for real numbers.

Rebuttal: the assumption that it is possible to find a suitable level of description of the universe means that we are able to define a suitable phase space, and so that we have identified the relevant observables, symmetries and laws for completely describing the universe. But this is the consequence of a deliberate and subjective act of the modeler, who defines what are the most pertinent and interesting parameters and symmetries to be used for defining the phase space. It is not an objective procedure [15]. This choice is historically dependent, as it is based on the universe observed by the modeler at a given time, and since the universe is non-ergodic and new things and functions come to exist continuously [3], it is not possible to define a complete phase space for the evolution of the universe once and for all. It is of course possible to provide an explanation a posteriori of an affordance, as we can explain heart once we observe and recognize it in an organism, but not the coming to existence of heart.

Objection Physicists always use math to devise models of the physical world and are well aware of their limitations: some features of the evolution of the biosphere can still be entailed, knowing that some approximations will be introduced and there will be some discrepancies between expected and actual results.

Rebuttal: in the case of affordances, we don't know the sample space for the reasons stated above, and therefore we cannot define a probability measure, nor can we define what is random, so we cannot estimate our error [16].

Objection We can always add to the model new objects and new relations.

Rebuttal: but still what can be deduced by the model cannot be novel, as it is already formalized. Semantics comes first, then comes syntax.

\section{Conclusion}

We are often pervaded by a profound sense of wonder when we observe the flourishing and creative power of the biosphere, and not seldom we are also amazed by the way animals and humans find creative solutions to problems or invent and build new tools. These phenomena are enabled by affordances, which also constantly change and appear. Since centuries we use mathematics to model phenomena of interest, and so provide explanations to understand what we observe or to control systems and processes. Some intrinsic limits of mathematical systems have been already raised in the Twentieth century, but mathematical models are still successfully applied in a plethora of cases. We would expect to be 
possible to formalize the evolution of the biosphere, to formally model affordances. Nevertheless, we face here an inherent limit: the properties of circularity and non-ergodicity characterizing affordances defeat any sound applicability of set theory, and consequently all math depending on it. In synthesis, we are claiming that the evolution of the biosphere, besides the impossibility of being entailed, is inherently not mathematizable: the world is not an algorithm. (Apologies to Pythagoras, Plato, Neoplatonists, Newton, Bohr, and ...) Starting from a discussion on the meaning of words in a language and moving to the foundation of mathematics, Wittgenstein had to some extent already contended that mathematical inferences do not bring new knowledge. In Remarks on the Foundation of Mathematics [26] he indeed maintains that surprise cannot come from an inference - and, if any surprise should come, it is simply because something was not yet understood. We are aware that our claim might be considered as a negative result that brings shadow and further limits the range of mathematical understanding of the reality. On the contrary, we believe that this incompleteness is a way to be creative [11] and achieve a deeper awareness of the world: "We are of Nature, not above Nature" [13].

\section{Epilogue}

But how many kinds of sentence are there? Say assertion, question and command? There are countless kinds; countless different kinds of use of all the things we call "signs", "words", "sentences". And this diversity is not something fixed, given once for all; but new types of language, new language-games, as we may say, come into existence, and others become obsolete and get forgotten. (We can get a rough picture of this from the changes in mathematics.)

(Wittgenstein, Philosophical investigations, first. publ. in 1953, 4th ed. 2009, WileyBlackwell, UK)

\section{References}

[1] J.L. Borges. The library of Babel. In Collected fictions. Penguin New York, 1998.

[2] C. Campbell, A. Olteanu, and K. Kull. Learning and knowing as semiosis: Extending the conceptual apparatus of semiotics. Sign Systems Studies, 47(3/4):352-381, 2019.

[3] M. Cortes, S.A. Kauffman, A.R. Liddle, and L. Smolin. Biocosmology: Biology from a cosmological perspective. To be published, 2021.

[4] C. Fernando, G. Kampis, and E. Szathmáry. Evolvability of natural and artificial systems. Procedia Computer Science, 7:73-76, 2011.

[5] J.J. Gibson. The senses considered as perceptual systems. Houghton Mifflin, 1966.

[6] S. Harnad. The symbol grounding problem. Physica D: Nonlinear Phenomena, 42(13):335-346, 1990. 
[7] L. Jamone, E. Ugur, A. Cangelosi, L. Fadiga, A. Bernardino, J. Piater, and J. SantosVictor. Affordances in psychology, neuroscience, and robotics: A survey. IEEE Transactions on Cognitive and Developmental Systems, 10(1):4-25, 2016.

[8] T. Jech. Set theory. Springer, the third millennium edition edition, 2006.

[9] I. Kant. Kritik of Judgement. Macmillan, 1892.

[10] S.A. Kauffman. Investigations. Oxford University Press, 2000.

[11] S.A. Kauffman. Humanity in a creative universe. Oxford University Press, 2016.

[12] S.A. Kauffman. A world beyond physics: the emergence and evolution of life. Oxford University Press, 2019.

[13] S.A. Kauffman. Eros and logos. Angelaki, 25(3):9-23, 2020.

[14] M.H. Le and D. Wang. Structure and membership of gut microbial communities in multiple fish cryptic species under potential migratory effects. Scientific reports, 10(1):1-12, 2020.

[15] G. Longo and M. Montévil. Perspectives on organisms: Biological time, symmetries and singularities. Springer, 2013.

[16] G. Longo, M. Montévil, and S.A. Kauffman. No entailing laws, but enablement in the evolution of the biosphere. In Proceedings of GECCO 2012 - The 14th Genetic and Evolutionary Computation Conference, pages 1379-1392, 2012.

[17] C.J. Lote. Principles of renal physiology, chapter 6, pages 70-85. Kluwer Acedemic Publishers, 4th edition, 2000.

[18] U.C. Merzbach and C.B. Boyer. A history of mathematics. John Wiley \& Sons, third edition, 2011.

[19] M. Montévil. Possibility spaces and the notion of novelty: from music to biology. Synthese, 196(11):4555-4581, 2019.

[20] G.H. Moore. Zermelo's axiom of choice: Its origins, development, and influence. Courier Corporation, 2012.

[21] L.A. Olsson, C.L. Nehaniv, and D. Polani. From unknown sensors and actuators to actions grounded in sensorimotor perceptions. Connection Science, 18(2):121-144, 2006.

[22] R. Pfeifer and J. Bongard. How the Body Shapes the Way We Think: A New View of Intelligence. MIT Press, Cambridge, MA, 2006. 
[23] A. Roli and S.A. Kauffman. Emergence of organisms. Entropy, 22(10):1163:1-12, 2020.

[24] C. Shannon. A mathematical theory of communication. The Bell System Technical Journal, 27(1,2):379-423,623-656, 1948.

[25] J. von Uexküll. A foray into the worlds of animals and humans: With a theory of meaning. University of Minnesota Press, 2010. Trad. by J.D. O'Neil. Originally published in German in 1934.

[26] L. Wittgenstein. Remarks on the foundations of mathematics. Blackwell, Oxford, UK, 1958. R. Rhees and G.E.M. Anscombe trans. 